\title{
Antibiogram-Derived Radial Decision Trees: An Innovative Approach to Susceptibility Data Display
}

\author{
${ }^{1}$ Rocco J. Perla and ${ }^{2}$ Paul P. Belliveau \\ ${ }^{1}$ Department of Clinical Microbiology and Infection Control, Health Alliance Hospitals \\ 60 Hospital Road, Leominster, MA 01453 \\ ${ }^{2}$ Department of Pharmacy Practice, Massachusetts College of Pharmacy and Health Sciences, Worcester
}

\begin{abstract}
Hospital antibiograms (ABGMs) are often presented in the form of large 2-factor (single organism vs. single antimicrobial) tables. Presenting susceptibility data in this fashion, although of value, does have limitations relative to drug resistant subpopulations. As the crisis of antimicrobial drug-resistance continues to escalate globally, clinicians need (1) to have access to susceptibility data that, for isolates resistant to first-line drugs, indicates susceptibility to second line drugs and (2) to understand the probabilities of encountering such organisms in a particular institution. This article describes a strategy used to transform data in a hospital ABGM into a probability-based radial decision tree (RDT) that can be used as a guide to empiric antimicrobial therapy. Presenting ABGM data in the form of a radial decision tree versus a table makes it easier to visually organize complex data and to demonstrate different levels of therapeutic decision-making. The RDT model discussed here may also serve as a more effective tool to understand the prevalence of different resistant subpopulations in a given institution compared to the traditional ABGM.
\end{abstract}

Key words: Antibiogram, decision-trees, interdisciplinary, susceptibility

\section{INTRODUCTION}

The Clinical and Laboratory Standards Institute (CLSI, formerly the National Committee for Clinical Laboratory Standards) defines an antibiogram (ABGM) as an overall profile of antimicrobial susceptibility results of a microbial species to a battery of antimicrobial agents ${ }^{[1]}$. Antibiograms have long been used as an epidemiological tool to characterize the susceptibility patterns and profiles of bacterial species over time in clinical settings and they are also believed to play an important role as a guide to empiric antimicrobial therapy ${ }^{[1]}$. Antibiograms are most often presented in the form of large tables that compare different organism-antimicrobial agent susceptibility combinations in a one-to-one correspondence. We believe that presenting susceptibility data in the form of a large 2-factor (single organism vs. single antimicrobial) table, although of value, does have limitations relative to drug-resistant subpopulations. As the problem and crisis of drug-resistant organisms continues to escalate in both hospital and community settings ${ }^{[2,3]}$, clinicians need (1) to have access to susceptibility data that, for isolates resistant to first-line drugs, indicates susceptibilities to second line drugs and (2) to understand the probabilities of encountering such organisms in a particular institution.

The purpose of this article, therefore, is to describe a strategy used to transform data in a hospital ABGM into a probability-based radial decision tree (RDT) that can be used as a guide to empiric antimicrobial therapy for the treatment of infectious diseases, with an emphasis on some of the most important resistance trends facing hospitals today. Presenting ABGM data in the form of a radial decision tree versus a table makes it easier to visually organize complex data and to demonstrate different levels of therapeutic decisionmaking. The RDT model discussed here may also serve as a more effective tool to understand the prevalence of different resistant phenotypes in a given institution compared to the traditional ABGM. Lastly, the RDT model developed here represents an interdisciplinary collaboration between microbiologists, pharmacists and infection control practitioners as it relates to the complex problem of antimicrobial resistance, an approach that is currently recommended by the infectious disease and infection control community ${ }^{[4,5]}$.

\section{MATERIALS AND METHODS}

Institution: Antibiogram data was collected from HealthAlliance Hospital, which is a medium-sized, nonurban, community hospital in Central Massachusetts, from December 2004 through August 2005. The HealthAlliance laboratory provides microbiology services for two long-term care facilities (LTCF) and outpatients. Along with in-patient susceptibility data, both LTCF resident and outpatient susceptibility data were included in the analysis because (i) increasing drug resistance is occurring in the community, (ii)

Corresponding Author: $\quad$ Dr. Rocco J. Perla, Department of Clinical Microbiology and Infection Control, Health Alliance Hospitals, 60 Hospital Road, Leominster, MA 01453, Tele: (978) 466-2064; Fax: (978) 466-2553 
many LTCF patients are regularly admitted to the hospital with positive culture results shortly after or shortly before admission and (iii) our aim was to produce results that were general and which could be broken down by different environments and subcategories during subsequent analyses.

Susceptibility testing: Susceptibility testing was performed using the Vitek Legacy automated susceptibility testing system (bioMerieux, Durham, $\mathrm{NC}$ ) that is based on the microdilution minimum inhibitory concentration (MIC) method ${ }^{[6]}$. Grampositive organisms were tested using the Vitek 109 Gram-Positive Susceptibility (GPS) card (bioMerieux, Durham, NC). Gram-negative organisms were tested using the Vitek 142 Gram-Negative Susceptibility (GNS) card (bioMerieux, Durham, NC).

Data collection and analysis: Antibiogram data was collected using the Vitek Data Trac reporting software (bioMerieux, Durham, NC). The NCCLS M39-A2 document was used as a guide to collect and analyze data during the ABGM development process ${ }^{[1]}$. The following data analysis recommendations of the M39A2 guidelines were followed: (i) inclusion of final and verified results only (ii) inclusion of only the first isolate of a given species encountered for a patient during the analysis period irrespective of body site and phenotypic characteristics (e.g., antimicrobial susceptibility pattern) (iii) inclusion of only organisms with 30 or more isolates tested (iv) inclusion of only diagnostic specimens (not surveillance or environmental cultures) (v) validation of software susceptibility test results by manual methods (vi) analysis and review of anomalous and highly improbable susceptibility test results. The susceptibility data collected in this study represents a nine-month period (December, 2004 through August, 2005). Although the M39-A2 recommends analyzing data during a 12-month period, this nine-month timeframe was selected because (i) prior to this nine-month period a different gram-positive card was in use, (ii) the current study is intended mainly for demonstration purposes and (iii) the sample sizes during this period were large enough for meaningful analysis of the data. The M39-A2 document also recommends reporting only the percentage of isolates that are susceptible to an antimicrobial agent. However, because level 2 decisions in the RDT (see below) stem from resistant microbial subpopulations, level 1 includes the percentage of isolates that are susceptible and resistant to level 1 agents, while level 2 includes only the percent susceptible. Strains with intermediate-level susceptibility were classified as resistant.

Decision tree: A subset of two species was selected from the ABGM susceptibility data to demonstrate the RDT model and concept. The two organisms include
Staphylococcus aureus $(\mathrm{n}=307)$ and Pseudomonas aeruginosa $(\mathrm{n}=66)$. These microbial species were selected because they are well documented as pathogens that have developed resistance to different classes of antimicrobials and are often associated with infections that are difficult to treat. It should be emphasized that the purpose of this article is to demonstrate the concept of an ABGM-derived RDT and not to address all clinically relevant findings revealed in the ABGM.

\section{RADIAL STRUCTURE AND DECISION TREES}

The radial structure of the antibiogram-derived decision tree addresses the fact that empiric (as well as culture-guided) antimicrobial therapy can have different starting points relative to the types of agents considered first-line and second-line therapy. Figure 1 demonstrates the basic structure of the RDT model. The center circle represents the microbial species in question. The first circular level around the microbial species (level 1) represents antimicrobials that are considered first-line agents or agents whose susceptibility should be considered during the initial selection process of an antimicrobial agent for that organism. For example, when treating a suspected or known $S$. aureus infection, the oxacillin susceptibility should be an initial concern that influences the selection of the initial agent.

The second level in the RDT (level 2) addresses therapeutic options if resistance to the first line agents is known or suspected. The degree to which one can reasonably suspect resistance to an antimicrobial agent empirically is largely a function of the susceptibility data reported in an ABGM. In the RDT, the susceptibility values (i.e., percent susceptible or resistant) are provided on the branches in the tree. Inasmuch as level 2 addresses resistant microbial subpopulations of a given species, this level represents the most difficult and challenging therapeutic situations. By multiplying the susceptibility percentages of any branch on the tree together, one can get an idea of the prevalence of a microbial susceptibility phenotype as it relates to more than one antibiotic. Additionally, if a clinician suspects resistance to a level 1 agent (i.e., based on patient location or history), the RDT allows for an educated prediction of susceptibility to level 2 agents for that resistant subpopulation. These pieces of information cannot be directly determined using traditional ABGMs.

Rationale for antibiotic choices in radial decision tree: The selection of specific antibiotics and their placement in the $S$. aureus or $P$. aeruginosa RDT are based on the interrelatedness of resistance mechanisms among different antibiotics, established practice guidelines and the peer reviewed literature in clinical microbiology and infectious diseases. 


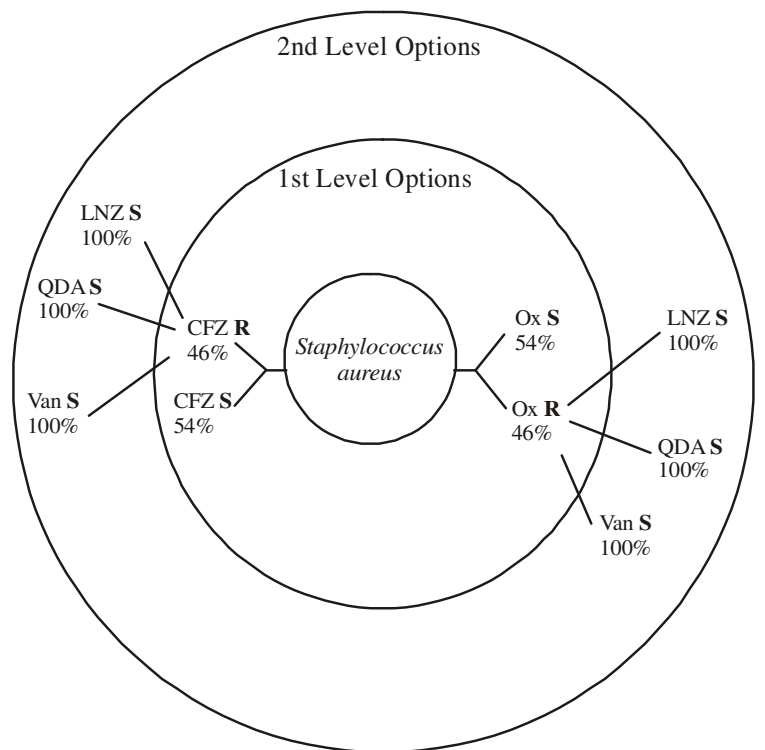

Fig. 1: Radial decision tree for S. aureus $(\mathrm{Ox}=\mathrm{oxacillin}$; $\mathrm{CFZ}=$ cefazolin; Van= Vancomycin; QDA= quinupristin/dalfopristin; $\quad$ LNZ=linezolid). $\mathrm{S}=$ Susceptible; $\mathrm{R}=$ Resistant

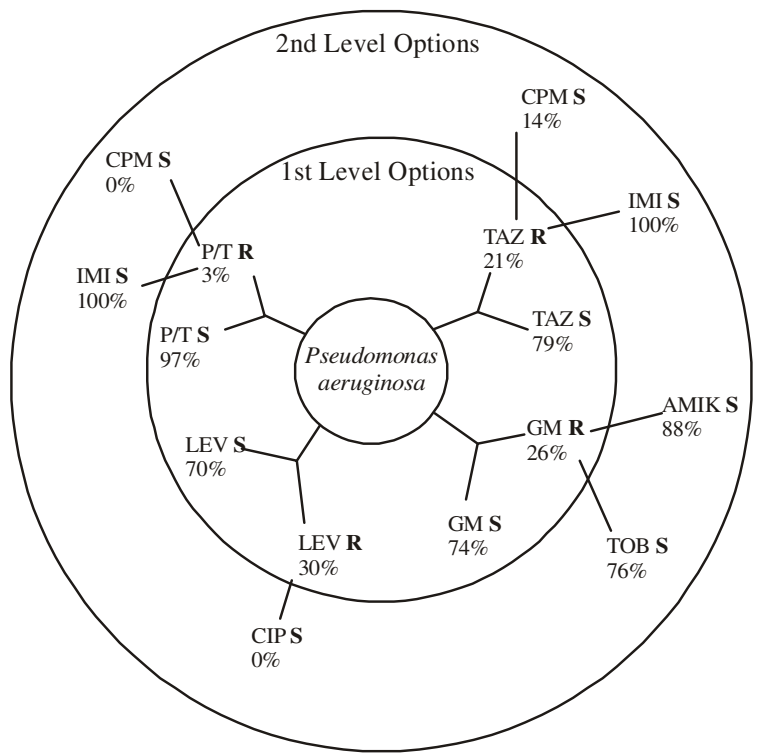

Fig. 2: Radial decision tree for $P$. aeruginosa (LEV=levofloxacin; GM=gentamicin; TAZ= ceftazidime; $\mathrm{P} / \mathrm{T}=$ piperacillin/tazobactam; $\mathrm{CIP}=$ ciprofloxacin; $\mathrm{TOB}=$ Tobramycin; $\mathrm{AMIK}=$ amikacin; IMI=imipenem; $\mathrm{CPM=cefepime).}$ $\mathrm{S}=$ Susceptible; $\mathrm{R}=$ Resistant

Consideration of each antibiotic in the RDT was based on the antibiotic's indication to treat infections typical for each pathogen. As with traditional antibiogram data, the RDT does not account for infection location, severity of infection, or other important patient-dependent factors (such as $\beta$-lactam allergies) that may affect treatment choices. A comprehensive technical description and justification of all antibiotic selections made in Fig. 1 and 2 is available from the corresponding author. However, it should be emphasized that our intention is to introduce a different and unique model for representing susceptibility data and that clinicians and epidemiologists at different institutions may chose different antibiotics in a particular RDT based on institutional variations in resistance trends and clinical experiences and preferences. In other words, our RDT model is one of many possible RDT models that may be developed and modified over time.

Practical utility of the RDT: The in vitro ( $a$ posteriori) probability of encountering an isolate with a specific phenotype can be calculated by multiplying the susceptibility values in the RDT. For example, the probability of encountering a $S$. aureus isolate that is oxacillin resistant and vancomycin susceptible in Fig. 1 is determined by the following calculation:

oxacillin resistance $(0.46) \mathrm{X}$ vancomycin susceptible $(1.0)=0.46$

Alternatively, there is little chance of encountering an oxacillin-resistant, vancomycin-resistant isolate (oxacillin resistance $(0.46) \mathrm{X}$ vancomycin resistant $(0)=0)$. While these examples provide information that is simple and somewhat intuitive (and does not necessarily require a calculation) since resistance to level 2 antibiotics in the $S$. aureus RDT is presently considered a rare event ${ }^{[7-10]}$, this calculation demonstrates the multiplicative probability susceptibility profiling that is a feature of the RDT (and is not addressed in traditional ABGMs).

The $P$. aeruginosa RDT is more complex from a therapeutic decision-making and in vitro susceptibilitytesting standpoint and may better demonstrate the value of multiplicative probability as it relates to susceptibility data. Utilizing the same type of calculation described with the $S$. aureus RDT, the probability of encountering a $P$. aeruginosa isolate that is ceftazidime resistant and imipenem susceptible is 0.21 . This could be compared to the probability of encountering a ceftazidime resistant, imipenem resistant isolate (probability of 0 ) or a ceftazidime susceptible isolate (0.79) to help assist with empiric treatment decisions. Alternatively, when choosing a second Gram-negative antibiotic to combine with ceftazidime, one could determine the probability of encountering an isolate that is: gentamicin susceptible (0.74); gentamicin resistant and amikacin susceptible (0.23); levofloxacin susceptible (0.70); or levofloxacin resistant and ciprofloxacin susceptible $(0)$.

\section{DISCUSSION}

The Radial Decision Tree model developed in this article addresses a number of important issues and 
concerns relative to antibiogram data that is routinely collected and analyzed in clinical microbiology laboratories and presented and disseminated hospitalwide. Below is a review of the benefits and limitations of the antibiogram-derived radial decision tree:

\section{Benefits of the radial decision tree}

* Reflects the logical thought process of antimicrobial selection that is influenced by knowledge of institution-specific resistance trends;

* Provides a quick visual "gestalt" of how clinically important drugs respond-and are likely to respond-to clinically significant microbial species;

* Provides a robust visual representation of complex data that could not be efficiently communicated in a traditional antibiogram without overcomplicating the display of information in the typical 2-factor table;

* Provides an opportunity to educate clinicians, pharmacists and microbiologists about logical empiric therapeutic decisions and resistance trends in their local environment;

* Provides additional epidemiological information regarding the prevalence of resistant microbial species to more than one agent at a time.

\section{Limitations of the radial decision tree}

* Like traditional antibiograms, the RDT provides an in vitro guide or reference point for the empiric treatment of infectious diseases;

* As with traditional antibiogram data, the RDT does not account for infection location, severity of infection, or other important patient-dependent factors (such as $\beta$-lactam allergies) that may affect treatment choices;

* The RDT developed and discussed here is not unit or location specific, but like traditional ABGMs the RDT could be constructed to reflect the susceptibility data and patterns in a particular unit or location;

* Like traditional ABGM data, the RDT data and structure is limited to the agents tested in the clinical microbiology laboratory.

\section{CONCLUSION}

It is important to emphasize that the increasing challenge and complexity of antimicrobial susceptibility testing, analysis and reporting requires the interdisciplinary collaboration and efforts of microbiologists, epidemiologists, clinicians and pharmacists in order to generate and communicate the most accurate and useful susceptibility data possible ${ }^{[1,4,5]}$. The RDT model developed here is an example of this type of interdisciplinary approach and one that we believe provides a relatively straightforward yet dynamic therapeutic guide and therapeutic decision-making learning tool.

\section{ACKNOWLEDGEMENTS}

Both authors contributed equally to this research. The authors thank Paul Concemi for critically reviewing a previous version of this article, Francis R. Landry for providing valuable assistance with the literature review and HealthAlliance Hospitals for their support.

\section{REFERENCES}

1. Clinical Laboratory and Standards Institute/NCCLS. Analysis and presentation of cumulative antimicrobial susceptibility test data; Approved guideline-second edition. CLSI document M39-A2, 2005. NCCLS, 940 West Valley Road, Suite 1400, Wayne Pennsylvania 19087-1898.

2. Levy, S.B. and T.F. O'Brien, 2005. Global antimicrobial resistance alerts and implications. Clin. Infect. Dis., 41: S219-20.

3. Chambers, H.F., 2005. Community-associated MRSA-Resistance and virulence converge. N. Engl. J. Med., 352: 1485-7.

4. Larson, E., L. Saiman, J. Haas, A. Neumann, F.D. Lowry, B. Fatato and S. Bakken, 2005. Perspectives on antimicrobial resistance: Establishing an interdisciplinary research approach. Am. J. Infect. Control, 33: 410-8.

5. Zapantis, A., M.K. Lace, R.T. Horvat, D. Grauer, B.J. Barnes, B. O'Neal and R. Couldry, 2005. Nationwide antibiogram analysis using NCCLS M39-A guidelines. J. Clin. Microbiol., 43: 262934.

6. Clinical Laboratory and Standards Institute/NCCLS. Performance standards for antimicrobial susceptibility testing; Fifteenth informational supplement. CLSI/NCCLS document M100-S15, 2005. Clinical Laboratory and Standards Institute, 940 West Valley Road, Suite 1400, Wayne Pennsylvania 19087-1898.

7. Jones, R.N., 2003. Global epidemiology of antimicrobial resistance among communityacquired and nosocomial pathogens: A five-year summary from the SENTRY antimicrobial surveillance program (1997-2001). Semin. Respir. Crit. Care Med., 24: 121-34.

8. Peeters, M.J. and J.C. Sarria, 2005. Clinical characteristics of linezolid-resistant Staphylococcus aureus infections. Am. J. Med. Sci., 330: 102-4.

9. Ruef, C., 2004. Epidemiology and clinical impact of glycopeptide resistance in Staphylococcus aureus. Infection, 32: 315-27.

10. Wilson, P., J.A. Andrews, R. Charlesworth, R. Walesby, M. Singer, D.J. Farrell and M. Robbins, 2003. Linezolid resistance in clinical isolates of Staphylococcus aureus. J. Antimicrobial Chemother., 51: 186-8. 